\title{
High Beta-Palmitate Formula and Bone Strength in Term Infants: A Randomized, Double-Blind, Controlled Trial
}

\author{
Ita Litmanovitz • Keren Davidson • Alon Eliakim • Rivka H. Regev • \\ Tzipora Dolfin · Shmuel Arnon · Fabiana Bar-Yoseph • \\ Amit Goren · Yael Lifshitz · Dan Nemet
}

Received: 11 June 2012/ Accepted: 18 October 2012/Published online: 20 November 2012

(C) Springer Science+Business Media New York 2012

\begin{abstract}
We aimed to compare the effect of 12-week feeding of commercially available infant formulas with different percentages of palmitic acid at $s n-2$ (beta-palmitate) on anthropometric measures and bone strength of term infants. It was hypothesized that feeding infants with high beta-palmitate (HBP) formula will enhance their bone speed of sound (SOS). Eighty-three infants appropriate for gestational age participated in the study; of these, 58 were formula-fed and 25 breast-fed infants, serving as a reference group. The formula-fed infants were randomly assigned to receive HBP formula ( $43 \%$ of the palmitic acid is esterified to the middle position of the glycerol backbone, study group; $n=30$ ) or regular formula with lowbeta palmitate (LBP, $14 \%$ of the palmitic acid is esterified to
\end{abstract}

F. Bar-Yoseph, A. Gorem, and Y. Lifshitz are employees of Enzymotec. All other authors have stated that they have no conflict of interest.

I. Litmanovitz $(\bowtie) \cdot$ R. H. Regev $\cdot$ T. Dolfin $\cdot$ S. Arnon

Department of Neonatology, Meir Medical Center,

59 Tchernichovsky Street, 44281 Kfar Saba, Israel

e-mail: litmani@clalit.org.il

I. Litmanovitz · A. Eliakim · R. H. Regev · T. Dolfin ·

S. Arnon - D. Nemet

Sackler Faculty of Medicine, Tel Aviv University,

69978 Tel Aviv, Israel

\section{K. Davidson}

School of Nutritional Sciences, The Hebrew University of Jerusalem, 76100 Rehovot, Israel

A. Eliakim - D. Nemet

Department of Pediatrics, Meir Medical Center,

59 Tchernichovsky Street, 44281 Kfar Saba, Israel

F. Bar-Yoseph · A. Goren · Y. Lifshitz

Enzymotec Ltd., Kfar Baruch, Israel the middle position of the glycerol backbone, $n=28$ ). Sixtysix infants completed the 12-week study. Anthropometric and quantitative ultrasound measurements of bone SOS for assessment of bone strength were performed at randomization and at 6 and 12 weeks postnatal age. At randomization, gestational age, birth weight, and bone SOS were comparable between the three groups. At 12 weeks postnatal age, the mean bone SOS of the HBP group was significantly higher than that of the LBP group $(2,896 \pm 133$ vs. $2,825 \pm 79 \mathrm{~m} / \mathrm{s}$ respectively, $P=0.049)$ and comparable with that of the breast-fed group $(2,875 \pm 85 \mathrm{~m} / \mathrm{s})$. We concluded that infants consuming HBP formula had changes in bone SOS that were comparable to those of infants consuming breast milk and favorable compared to infants consuming LBP formula.

Keywords Beta-palmitate - Bone speed of sound . Infant formula $\cdot$ Quantitative ultrasound

\section{Introduction}

During the last decade, substantial efforts were made to determine the factors that influence bone mineral accretion in healthy children. This arises from the notion that osteoporosis originates in childhood [1]. Providing optimal nutrition in childhood may be essential in our effort to reach the highest possible peak bone mass. The absorption of nutrients, such as minerals, fats, carbohydrates, and proteins, is significantly important for normal infant growth and development and may contribute to early bone mineral accretion [2].

In human breast milk (BM) and in most infant formulas, about $50 \%$ of the dietary calories are supplied as fat $[3,4]$. Palmitic acid, comprises $17-25 \%$ of fatty acids in BM, of which $70-75 \%$ is esterified to the $s n-2(\beta)$ position of the triglyceride [3]. Previous studies have shown that to assure 
optimal fat absorption, palmitic acid is best absorbed from human milk as $s n-2$ monoacylglycerol $[5,6]$ and is conserved as such through digestion, absorption, and chylomicron triacylglycerol synthesis [7]. In contrast, the free palmitic acid originating from the -1 and -3 positions of vegetable oils, commonly used in manufactured infant formulas [8], has high tendency to create complexes with dietary minerals such as calcium to form fatty acid soaps $[9,10]$, resulting in loss of both calcium and fatty acids in the stool. Beta-palmitate is a fat ingredient that mimics the fatty acids positioned in BM. Previous studies have shown that its use in both term and preterm infant formulas may enhance fatty acids and calcium absorption [8, 11-14].

Quantitative ultrasound (QUS) measurements of bone speed of sound (SOS) is now considered an important tool for the diagnosis and follow-up of bone strength in term and preterm infants [15]. QUS measurements of bone are noninvasive, painless, portable, and relatively inexpensive, involving no ionizing radiation and posing no known adverse effects. In addition to bone mineral density (BMD), it measures bone cortical thickness, elasticity, and microarchitecture and provides a more complete picture of bone strength in adults, children, and newborns [16-25].

The aim of the present study was to assess the short term effect of consuming high-beta-palmitate (HBP) formula compared with regular infant formula, comprising lowbeta-palmitate (LBP), on bone strength of term newborns as the primary outcome and on anthropometric measures as the secondary outcome. We hypothesized that compared with LBP formula, feeding term newborns with HBP formula will enhance their bone strength.

\section{Methods}

\section{Study Design and Participants}

Healthy term (>37 weeks) infants, appropriate for gestational age and younger than 14 days of age, were eligible for entry to this randomized, double-blind controlled, longitudinal trial. Infants were excluded if they had congenital or chromosomal disorder or if noncompliance with the trial feeding regimen was expected of their parents. Infants were enrolled only if their mother unequivocally decided to formula feed within the first 2 weeks of the baby's life. By means of an automatic randomization system, infants were randomly assigned to receiving HBP formula (forming the study group) or LBP formula (regular infant formula; forming the control group). Formulas were packed in identical and unmarked boxes, with the personnel caring for the infants and mothers being blinded to their content.

Infant and maternal demographic characteristics for the HBP and LBP formula groups are listed in Table 1.
Included was also a gestational age matched reference group of term infants consuming BM. Twins were initially assigned to the same group; however, to avoid possible genetic or intrauterine effects, only the twin with the larger birth weight was included in the analysis. The infants were followed up twice, at 6 and 12 weeks.

The study was conducted according to the principles of the Declaration of Helsinki and good clinical practice. The protocol was approved by the ethics committee of Meir Medical Center, Kfar Saba, Israel, and by the Israeli Ministry of Health. Before enrollment, all parents signed a written informed consent.

\section{Formula-Feeding Regimen}

We used currently available commercial formulas. Both HBP and LBP formulas were produced by the same manufacturing company (Materna Laboratories, Kibbutz Maabarot, Israel) and under the same conditions using ingredients such as minerals and vitamins from the same batches, thus resulting in identical commercial formulas (standard vegetable oil mix of palm kernel oil, rapeseed oil, sunflower oil, and palm oil or structured palm oil) that differ mainly in the palmitic structural distribution (14 and $43 \%$ of the palmitic acid esterified to the midpoint position of the glycerol backbone, respectively) (InFat ${ }^{\circledR}$ Advanced Lipids AB, Migdal HaEmeq, Israel; Table 2). Infants were fed ad libitum; no supplementary feeding was provided. All infants, including BM infants, received $400 \mathrm{IU} /$ day of vitamin $\mathrm{D}$ during the study intervention period.

\section{Anthropometric Measurements}

Measurements of growth and bone SOS were done at randomization and at 6 and 12 weeks postnatal age by a single trained technician, who was blinded to the study group assignment. The measurements covered the following variables: body weight (the mean of three measurements; Model 20 Tabletop Infant Scale, Olympic Medical, Seattle, WA), body length (the mean of two measurements of recumbent crown-heel length to the nearest $0.1 \mathrm{~cm}$; O'Leary Preemie LengthBoard, Ellard Instrumentation Ltd., Monroe, WA), and fronto-occipital head circumference (standard 1-cm wide measuring tape to the nearest $0.1 \mathrm{~cm}$ ).

\section{QUS Measurements of Bone SOS}

The left tibial SOS was measured by the QUS (Sunlight Premier Software, Omnisense 7000/8000, BeamMed Ltd., Petah Tikva, Israel), a method designed to measure SOS at multiple skeletal sites by axial transmission. The measurement is based on the fact that ultrasound waves propagate faster through bone than through soft tissue. A standardized 
Table 1 Demographic characteristics of infants and mothers
Data are presented as mean \pm SEM

$H B P$ high beta-palmitate, $L B P$ low-beta palmitate, $B M$ human breast milk, SEM standard error of the mean

$* P<0.05$ compared with the $\mathrm{BM}$ group

\begin{tabular}{llll}
\hline Characteristic & Formula & BM $(n=25)$ \\
\cline { 3 - 4 } & HBP $(n=26)$ & LBP $(n=25)$ & \\
\hline Infant & & & \\
Gestational age (weeks) & $39.4 \pm 0.2$ & $39.2 \pm 0.2$ & $39.7 \pm 0.2$ \\
Type of delivery (vaginal, \%) & 76.0 & 64.0 & 68.0 \\
Twins (\%) & $15.4^{*}$ & 12.0 & 0.0 \\
Gender (male, \%) & 57.7 & $40.0^{*}$ & 68.0 \\
Birth weight (kg) & $3.28 \pm 0.1$ & $3.26 \pm 0.1$ & $3.38 \pm 0.1$ \\
Age at inclusion (days) & $3.3 \pm 0.5$ & $3.5 \pm 0.6$ & $3.2 \pm 0.4$ \\
Mother & & & $31.6 \pm 0.8$ \\
Maternal age (years) & $32.3 \pm 0.7$ & $33.3 \pm 0.8$ & 92.0 \\
Maternal education (>12 years) $(\%)$ & $65.4^{*}$ & $56.0^{*}$ & 32.0 \\
Primigravida (\%) & 15.4 & 16.0 & 0.0 \\
Maternal smoking (\%) & $15.4^{*}$ & $32.0 *$ & \\
\hline
\end{tabular}

Table 2 Composition of HBP and LBP formulas

\begin{tabular}{|c|c|c|}
\hline Composition & $\begin{array}{l}\text { Per } 100 \text { g powder of: } \\
\text { HBP }\end{array}$ & LBP \\
\hline Energy (kcal) & 510 & 510 \\
\hline Protein (lactalbumin/casein, 60/40) (g) & 12 & 12 \\
\hline Carbohydrate (g) & 55 & 55.7 \\
\hline Fat $(\mathrm{g})$ & 26 & 26 \\
\hline Calcium $(\mathrm{mg})^{\mathrm{a}}$ & 430 & 420 \\
\hline Vitamin D $(I U)^{a}$ & 372 & 404 \\
\hline \multicolumn{3}{|l|}{ Percentage by weight of total fatty acids ${ }^{b}$} \\
\hline $8: 0$ & 0.9 & 3 \\
\hline $10: 0$ & 0.8 & 2.2 \\
\hline $12: 0$ & 10.4 & 9.4 \\
\hline $14: 0$ & 4.3 & 4.2 \\
\hline $16: 0$ & 22 & 19 \\
\hline $18: 0$ & 4.4 & 6.4 \\
\hline $18: 1 n-9$ & 38.5 & 34.4 \\
\hline $18: 2 n-6$ & 14 & 15.1 \\
\hline $18: 3 n-3$ & 1.5 & 1.5 \\
\hline $20: 4$ & 0.42 & 0.53 \\
\hline $22: 6$ & 0.22 & 0.29 \\
\hline Other fatty acids & 2.9 & 5.1 \\
\hline 16:0 in $s n-2$ position $^{c}$ & 44 & 14 \\
\hline
\end{tabular}

$H B P$ high beta-palmitate, $L B P$ low-beta palmitate

${ }^{a}$ Value differences between the formulas are within analytical method deviation

b Included vegetable oil mix

c The ratio is normalized per position and calculated as percentage of $s n-2$ palmitic/3: \% total palmitic acid $\times 100$

procedure was followed, and the probe was placed on the left tibia at half the measured distance between the apex of the heel and the distal patellar apex. After calibrating the machine with a standard phantom, three measurements were obtained from the same site and the mean value calculated.
Measurement accuracy was $0.25-0.5 \%$ with a root mean square coefficient of variation of $0.4-0.8 \%$.

The measurements were performed by the same technician, who was blinded to group assignment. Formula consumption before each visit was based on a structured diary completed by the parents, calculating the mean total amount of formula feeds per kilogram of body weight over a 3-day period.

\section{Statistical Analysis}

Baseline characteristics of mothers and infants of the HBP and LBP formula groups were compared by pairwise $t$ test for scale outcomes and pairwise $\chi^{2}$ test for nominal outcomes. The mean SOS and primary study endpoint of the groups was tested with the analysis of covariance (ANCOVA) adjusted for infant birth weight. The model was run for baseline, 6-, and 12-week SOS measurements, once for available observations at each time point and once restricted to the subset of newborns, thus resulting in a complete set of SOS measurements. Serving as a reference group, all parameters of the BM group were also compared with that of the two formula groups. The similarity of the SOS measurements of the HBP formula group with those of the BM group was demonstrated by showing that the mean difference and the $95 \%$ confidence interval (CI) of both groups were close to zero.

Statistical analysis was conducted by SPSS software, version 17.0 (SPSS Inc., Chicago, IL). Data were expressed as mean \pm SEM. Statistical significance was inferred at 2 tails $P<0.05$. Power calculation indicated that a sample of about 20 infants in each arm could detect statistical significance, showing a difference of $80 \mathrm{~m} / \mathrm{s}$ in the SOS measurements with an $80 \%$ value for power. 


\section{Results}

A total of 83 term infants were enrolled. Twenty-five were exclusively breast-fed. Of the 58 formula-fed infants, 30 were randomly assigned to receive HBP formula and 28 LBP formula. The attrition rate by the end of the study period was $21 \%$ and was equally distributed between the three groups (Fig. 1). The sample of $21 \%$ of those who did not complete the study was matched with those who did according to gestational age and birth weight (data not shown).

Infant characteristics are presented in Table 1 . No significant differences were observed between the two formula groups. The BM group had a higher incidence of males than the LBP group (68 and $40 \%$, respectively) and included no twins. Infant growth for weight, length, weight gain, and head circumference are listed in Table 3.

Maternal characteristics of the two formula groups were comparable. Breast-feeding mothers had a significantly higher education level ( $>12$ years) and lower smoking rate during pregnancy compared with the mothers of the HBP and LBP formula groups (Table 1). However, the differences in maternal smoking did not significantly affect the infants' birth anthropometric measures and/or baseline SOS.

Anthropometric data during study visits at baseline and at 6 and 12 postnatal weeks showed no significant differences between the two formula groups. There was also no significant difference in formula consumption between these groups at 6 weeks (177 vs. $178 \mathrm{ml} / \mathrm{kg} / \mathrm{day}$, respectively) and 12 weeks (143 vs. $139 \mathrm{ml} / \mathrm{kg} /$ day $)(P>0.05$ for all).

At randomization, bone SOS was comparable for all three groups of the study. When compared with baseline, it was shown that bone SOS decreased significantly at 6 and 12 weeks postnatal age $(P<0.001)$. Although there were no significant differences between the groups at 6 weeks postnatal age, at 12 weeks postnatal age, the mean SOS of

Fig. 1 Participant flow diagram

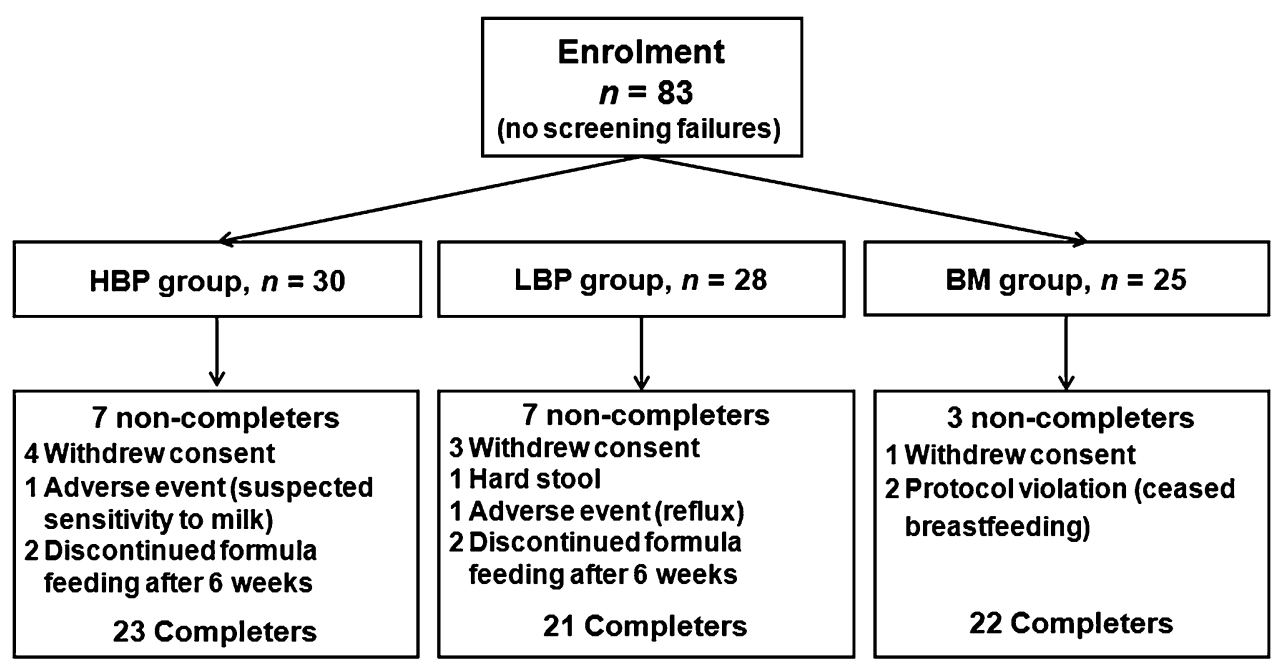

Table 3 Anthropometric parameters at baseline, 6, and 12 weeks postnatal age

\begin{tabular}{|c|c|c|c|c|c|}
\hline Time point & Formula & Weight $(\mathrm{kg})$ & Length $(\mathrm{cm})$ & Weight gain $(\mathrm{g} / \text { day })^{\mathrm{a}}$ & Head circumference $(\mathrm{cm})$ \\
\hline \multirow{3}{*}{ Baseline } & HBP & $3.2 \pm 0.1$ & $49.9 \pm 0.5$ & NA & $34.3 \pm 0.3$ \\
\hline & LBP & $3.1 \pm 0.1$ & $49.2 \pm 0.4$ & NA & $34.2 \pm 0.3$ \\
\hline & $\mathrm{BM}$ & $3.2 \pm 0.1$ & $50.3 \pm 0.5$ & NA & $34.9 \pm 0.3$ \\
\hline \multirow[t]{3}{*}{6 weeks } & HBP & $4.7 \pm 0.1$ & $55.7 \pm 0.4$ & $37.2 \pm 1.8$ & $37.6 \pm 0.3 *$ \\
\hline & LBP & $4.7 \pm 0.1$ & $55.2 \pm 0.5^{*}$ & $36.7 \pm 1.7$ & $37.4 \pm 0.4^{*}$ \\
\hline & $\mathrm{BM}$ & $5.0 \pm 0.1$ & $56.7 \pm 0.6$ & $37.2 \pm 1.8$ & $38.3 \pm 1.0$ \\
\hline \multirow[t]{3}{*}{12 weeks } & HBP & $6.0 \pm 0.2$ & $60.1 \pm 0.7$ & 29.31 .8 & $39.6 \pm 0.4$ \\
\hline & LBP & $5.9 \pm 0.2$ & $59.9 \pm 0.5$ & 26.81 .6 & $40.1 \pm 0.3$ \\
\hline & $\mathrm{BM}$ & $6.2 \pm 0.2$ & $60.9 \pm 0.4$ & 27.21 .4 & $39.9 \pm 0.4$ \\
\hline
\end{tabular}

Data are presented as mean \pm SEM

$H B P$ high beta-palmitate, $L B P$ low-beta palmitate, $B M$ human breast milk, $N A$ not applicable

$* P<0.05$ compared with the BM group

${ }^{\text {a }}$ Baseline to 6 weeks and 6-12 weeks 
Table 4 Bone SOS measurements at baseline, 6, and 12 weeks postnatal age

\begin{tabular}{llllll}
\hline Time point & HBP $(n=20)$ & LBP $(n=18)$ & BM $(n=22)$ & HBP vs. BM difference $(95 \%$ CI $)$ & HBP vs. LBP difference (95 \% CI) \\
\hline Baseline & $3,027 \pm 20$ & $3,001 \pm 22$ & $3,023 \pm 21$ & $1.3(-72.1,74.6)$ & $21.7(-49.2,92.6)$ \\
6 weeks & $2,920 \pm 24$ & $2,852 \pm 24$ & $2,915 \pm 26$ & $13.4(-65.1,92.0)$ & $68.6(-5.9,149.1)$ \\
12 weeks & $2,896 \pm 30^{* *}$ & $2,825 \pm 19$ & $2,875 \pm 18$ & $33.2(-36.6,103)$ & $74.7(0.33,149)^{*}$
\end{tabular}

Data are presented as mean \pm SEM of unadjusted SOS $(\mathrm{m} / \mathrm{s})$ or as $95 \%$ CI for group differences according to ANCOVA adjusted for birth weight

$S O S$ speed of sound, $H B P$ high beta-palmitate, $L B P$ low-beta palmitate, $B M$ human breast milk, $C I$ confidence interval, $S E M$ standard error of the mean

* Group differences statistically significant at $P<0.05$

** Significantly different from controls according to ANCOVA adjusted for birth weight at $P<0.05$

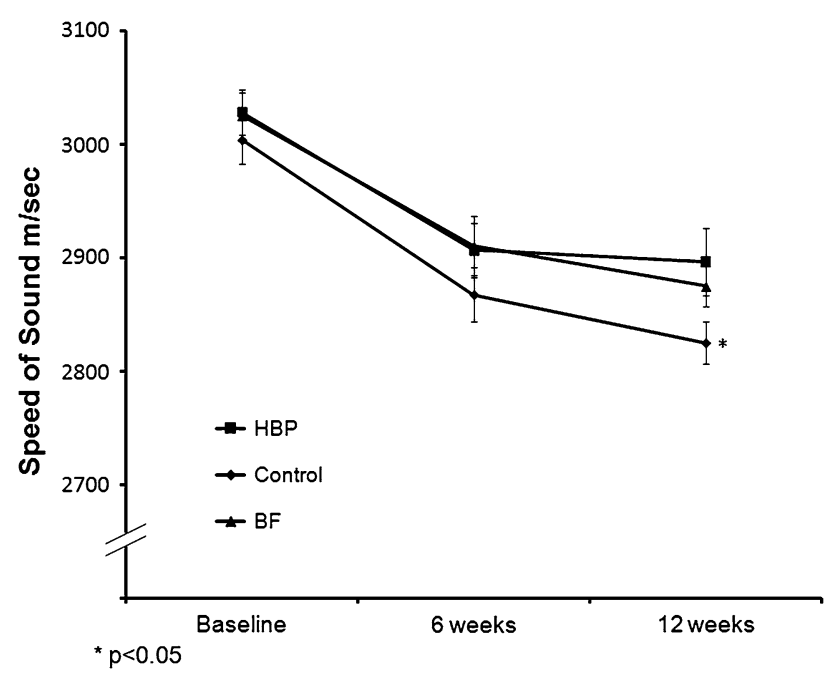

Fig. 2 Bone SOS at baseline and after 6 and 12 weeks in the 3 study groups. At 12 weeks, bone SOS in the HBP group was significantly higher than that in the LBP group

the HBP group was significantly higher than that of the LBP group (2,896 \pm 133 vs. $2,825 \pm 79 \mathrm{~m} / \mathrm{s}$, respectively, $P=0.049)$ and similar to that of the BM group $(2,875 \pm 85 \mathrm{~m} / \mathrm{s}, P=0.3$; Table 4 ; Fig. 2). The difference between the HBP and the LBP groups remained statistically significant when weight at each visit was used as a covariate in the ANCOVA $(P<0.05)$.

There was a twofold difference in maternal smoking between the two formula groups. This difference did not reach statistical significance. Further analysis with maternal smoking used as a covariant was not found to affect the change in bone SOS.

\section{Discussion}

As hypothesized in this randomized, controlled, doubleblind study, bone SOS of term newborns fed HBP formula was significantly higher than that of newborns fed LBP and comparable with that of term newborns fed BM. These data are consistent with two other studies that used dual-energy $\mathrm{X}$-ray absorptiometry (DEXA) that assessed bone mineralization [8, 26]. Kennedy et al. [8] showed in their randomized controlled trial that infants fed a greater proportion of palmitate in the $s n-2$ position have higher body bone mass at 12 weeks. In a complementary longitudinal study, term infants ingesting formula with palmitate in the $s n-1$ and $s n-3$ positions, developed reduced total body bone mineral content (BMC) compared with infants ingesting infant formula without palm oils [26]. Clearly, bone QUS and DEXA modalities are based on different measuring principles. Although DEXA mainly measures quantitative aspects of BMD, QUS relates to qualitative factors, such as bone elasticity, microarchitecture, geometry, and porosity of cortical bone that contribute to bone strength. We found that bone SOS of the HBP group was comparable with that of the BM group, again consistent with Kennedy et al. [8]. Studies in the 1990s published conflicting data regarding BMC of breast-fed versus formula-fed infants. Specker et al. [27] reported lower total body BMC in exclusively breast-fed infants compared with formula-fed infants. In contrast, others found comparable BMC with different types of feeding [28, 29]. However, the composition of other nutrients, especially fat composition, was not addressed in these early studies.

Our SOS measurements show a decrease in SOS in the first 12 weeks postnatal age for all infants regardless of the type of feeding. This is in agreement with studies in both preterm infants [21, 22] and term infants demonstrating a decrease in SOS [30] and DEXA [31]. The reasons for this phenomenon are not clear. It was suggested that the decline in BMD in healthy newborns is associated with a relative physiological decrease of the cortical area and the redistribution of bone tissue from the endocortical to the periosteal surface rather than with bone loss [30]. It is also possible that this decrease represents a delay between rapid bone linear growth and mineralization. In a nonrandomized clinical study, Zuccotti et al. [32] showed that at 4 months there is already increase in SOS, with no significant differences between exclusive breast-fed or formula-fed 
infants after 4 and 12 months. This is in agreement with our findings that HBP infants have comparable SOS values with breast-fed infants, yet it is in contrast to our finding that BM infants have higher SOS values than control infants. Of note is that although the authors compared between formula and BM feeding, they did not differentiate between the different types of formula milk. Further, given that bone SOS was done at 4 months, it is possible that the initial decline was missed.

To our knowledge, our study is the first to demonstrate bone status by using QUS for term infants consuming HBP or LBP formula. The measurements obtained by QUS may not only be related to BMD but also to additional parameters of bone strength and quality. Nevertheless, the study has several limitations. First, we had a relatively small sample size; however, there was adequate statistical power to detect differences between the two formula arms of the trial. A second limitation is a potential noncomleters bias. In this respect, we stress that the sample of $21 \%$ of those who did not complete the study was matched with those who did for gestational age and birth weight. Third, no longer-term follow-up after 12 weeks postnatal age was provided to examine the effect of supplementation of BM and HBP and LBP formulas on future bone strength. Thus, it is unclear whether the differences we found in bone SOS at infancy affect bone strength at older ages. Moreover, it is possible that the initial decrease in bone SOS and mineralization is a physiologic phenomenon that is necessary for bone development later in life. If this is the case, efforts to prevent this decrease are questionable. It should be emphasized that the findings of the HBP group was similar to those of the BM group and thus mimic a gold standard. Still, further studies are needed to elucidate the complex relationship between neonatal bone strength and the development of osteopenia and osteoporosis later in life.

In conclusion, at 12 weeks postnatal age, bone SOS of term infants consuming infant formula enriched with HBP was higher than that of infants consuming LBP formula, and comparable with that of the BM infants.

Acknowledgments We thank Prof. Raanan Shamir from the Institute of Gastroenterology, Nutrition and Liver Diseases, Schneider Children's Medical Center of Israel, for scientific support and Prof. Virginia A. Stallings from the Division of Gastroenterology, Hepatology and Nutrition, Children's Hospital, Philadelphia, for critical review. We also thank Diklah Geva from IntegriStat, Biostatistics Services, Tel Aviv, for the statistical support.

\section{References}

1. Janz K (2002) Physical activity and bone development during childhood and adolescence. Implications for the prevention of osteoporosis. Minerva Pediatr 54:93-104
2. Specker B (2004) Nutrition influences bone development from infancy through toddler years. J Nutr 134:691S-695S

3. Breckenridge WC, Marai L, Kuksis A (1969) Triglyceride structure of human milk fat. Can J Biochem 47:761-769

4. Giovannini M, Riva E, Agostoni C (1995) Fatty acids in pediatric nutrition. Pediatr Clin North Am 42:861-877

5. Tomarelli RM, Meyer BJ, Weaber JR, Bernhart FW (1968) Effect of positional distribution on the absorption of the fatty acids of human milk and infant formulas. J Nutr 95:583-590

6. Innis SM, Dyer R, Nelson CM (1994) Evidence that palmitic acid is absorbed as $s n-2$ monoacylglycerol from human milk by breast-fed infants. Lipids 29:541-545

7. Nelson CM, Innis SM (1999) Plasma lipoprotein fatty acids are altered by the positional distribution of fatty acids in infant formula triacylglycerols and human milk. Am J Clin Nutr 70:62-69

8. Kennedy K, Fewtrell MS, Morley R, Abbott R, Quinlan PT, Wells JC, Bindels JG, Lucas A (1999) Double-blind, randomized trial of a synthetic triacylglycerol in formula-fed term infants: effects on stool biochemistry, stool characteristics, and bone mineralization. Am J Clin Nutr 70:920-927

9. Small DM (1991) The effects of glyceride structure on absorption and metabolism. Annu Rev Nutr 11:413-434

10. Quinlan PT, Lockton S, Irwin J, Lucas AL (1995) The relationship between stool hardness and stool composition in breast- and formula-fed infants. J Pediatr Gastroenterol Nutr 20:81-90

11. Carnielli VP, Luijendijk IH, van Goudoever JB, Sulkers EJ, Boerlage AA, Degenhart HJ, Sauer PJ (1995) Feeding premature newborn infants palmitic acid in amounts and stereoisomeric position similar to that of human milk: effects on fat and mineral balance. Am J Clin Nutr 61:1037-1042

12. Carnielli VP, Luijendijk IH, Van Goudoever JB, Sulkers EJ, Boerlage AA, Degenhart HJ, Sauer PJ (1996) Structural position and amount of palmitic acid in infant formulas: effects on fat, fatty acid, and mineral balance. J Pediatr Gastroenterol Nutr 23:553-560

13. Lopez-Lopez A, Castellote-Bargallo AI, Campoy-Folgoso C, Rivero-Urgel M, Tormo-Carnice R, Infante-Pina D, Lopez-Sabater MC (2001) The influence of dietary palmitic acid triacylglyceride position on the fatty acid, calcium and magnesium contents of at term newborn faeces. Early Hum Dev 65(Suppl):S83-S94

14. Lucas A, Quinlan P, Abrams S, Ryan S, Meah S, Lucas PJ (1997) Randomised controlled trial of a synthetic triglyceride milk formula for preterm infants. Arch Dis Child Fetal Neonatal Ed 77: F178-F184

15. Rack B, Lochmuller EM, Janni W, Lipowsky G, Engelsberger I, Friese K, Kuster H (2012) Ultrasound for the assessment of bone quality in preterm and term infants. J Perinatol 32:218-226

16. Prins SH, Jorgensen HL, Jorgensen LV, Hassager C (1998) The role of quantitative ultrasound in the assessment of bone: a review. Clin Physiol 18:3-17

17. Mehta SS, Antich PP, Daphtary MM, Bronson DG, Richer E (2001) Bone material ultrasound velocity is predictive of whole bone strength. Ultrasound Med Biol 27:861-867

18. Zadik Z, Price D, Diamond G (2003) Pediatric reference curves for multi-site quantitative ultrasound and its modulators. Osteoporos Int 14:857-862

19. Nemet D, Dolfin T, Wolach B, Eliakim A (2001) Quantitative ultrasound measurements of bone speed of sound in premature infants. Eur J Pediatr 160:736-740

20. Eliakim A, Nemet D, Friedland O, Dolfin T, Regev RH (2002) Spontaneous activity in premature infants affects bone strength. J Perinatol 22:650-652

21. Litmanovitz I, Dolfin T, Friedland O, Arnon S, Regev R, Shainkin-Kestenbaum R, Lis M, Eliakim A (2003) Early physical activity intervention prevents decrease of bone strength in very low birth weight infants. Pediatrics 112:15-19 
22. Rubinacci A, Moro GE, Boehm G, De Terlizzi F, Moro GL, Cadossi R (2003) Quantitative ultrasound for the assessment of osteopenia in preterm infants. Eur J Endocrinol 149:307-315

23. Pereda L, Ashmeade T, Zaritt J, Carver JD (2003) The use of quantitative ultrasound in assessing bone status in newborn preterm infants. J Perinatol 23:655-659

24. Foldes AJ, Rimon A, Keinan DD, Popovtzer MM (1995) Quantitative ultrasound of the tibia: a novel approach for assessment of bone status. Bone 17:363-367

25. Kang C, Speller R (1998) Comparison of ultrasound and dual energy X-ray absorptiometry measurements in the calcaneus. Br J Radiol 71:861-867

26. Koo WW (2004) Reduced bone mineralization in infants fed palm olein-containing formula: a randomized, double-blinded, prospective trial. author reply. Pediatrics 114:899-900

27. Specker BL, Tsang RC, Hollis BW (1985) Effect of race and diet on human-milk vitamin D and 25-hydroxyvitamin D. Am J Dis Child 139:1134-1137

28. Park MJ, Namgung R, Kim DH, Tsang RC (1998) Bone mineral content is not reduced despite low vitamin $\mathrm{D}$ status in breast milk-fed infants versus cow's milk based formula-fed infants. J Pediatr 132:641-645

29. Hillman LS (1988) Bone mineral content in term infants fed human milk, cow milk-based formula, or soy-based formula. J Pediatr 113:208-212

30. Liao X, Zhang W, He J, Sun J, Huang P (2005) Bone measurements of infants in the first 3 months of life by quantitative ultrasound: the influence of gestational age, season, and postnatal age. Pediatr Radiol 35:847-853

31. Rauch F, Schoenau E (2001) Changes in bone density during childhood and adolescence: an approach based on bone's biological organization. J Bone Miner Res 16:597-604

32. Zuccotti G, Vigano A, Cafarelli L, Pivetti V, Pogliani L, Puzzovio M, Mora S (2011) Longitudinal changes of bone ultrasound measurements in healthy infants during the first year of life: influence of gender and type of feeding. Calcif Tissue Int 89: $312-317$ 